\title{
Subjective and Objective Appraisal of Activity in Adults with Obstructive Sleep Apnea
}

\author{
Eileen R. Chasens, ${ }^{1}$ Susan M. Sereika, ${ }^{1}$ Martin P. Houze, ${ }^{1}$ and Patrick J. Strollo ${ }^{2}$ \\ ${ }^{1}$ School of Nursing, University of Pittsburgh, 3500 Victoria Street, Pittsburgh, PA 15261, USA \\ ${ }^{2}$ School of Medicine, University of Pittsburgh, 3500 Victoria Street, Pittsburgh, PA 15261, USA \\ Correspondence should be addressed to Eileen R. Chasens, chasense@pitt.edu
}

Received 1 September 2010; Accepted 15 November 2010

Academic Editor: Iris Reuter

Copyright (c) 2011 Eileen R. Chasens et al. This is an open access article distributed under the Creative Commons Attribution License, which permits unrestricted use, distribution, and reproduction in any medium, provided the original work is properly cited.

\begin{abstract}
Objective. This study examined the association between obstructive sleep apnea (OSA), daytime sleepiness, functional activity, and objective physical activity. Setting. Subjects $(N=37)$ being evaluated for OSA were recruited from a sleep clinic. Participants. The sample was balanced by gender (53\% male), middle-aged, primarily White, and overweight or obese with a mean BMI of $33.98(\mathrm{SD}=7.35$; median BMI $=32.30)$. Over $40 \%$ reported subjective sleepiness (Epworth Sleepiness Scale $(\mathrm{ESS}) \geq 10)$ and had OSA $(78 \%$ with apnea + hypopnea index (AHI) $\geq 5 / \mathrm{hr})$. Measurements. Evaluation included questionnaires to evaluate subjective sleepiness (ESS) and functional outcomes (Functional Outcomes of Sleep Questionnaire (FOSQ)), an activity monitor, and an overnight sleep study to determine OSA severity. Results. Increased subjective sleepiness was significantly associated with lower scores on the FOSQ but not with average number of steps walked per day. A multiple regression analysis showed that higher AHI values were significantly associated with lower average number of steps walked per day after controlling patient's age, sex, and ESS. Conclusion. Subjective sleepiness was associated with perceived difficulty in activity but not with objectively measured activity. However, OSA severity was associated with decreased objective physical activity in aging adults.
\end{abstract}

\section{Introduction}

The increased prevalence of persons in the USA who are overweight or obese has been accompanied by a parallel increase in the prevalence of obstructive sleep apnea (OSA) [1]. Data from multiple studies suggest that excessive body weight is a primary risk factor for type 2 diabetes, cardiovascular disease, and OSA [2-5]. Increased physical activity has significant health benefits that include not only improved weight control but also an independent reduction in the risk for diabetes, hypertension, cardiovascular disease, depression, and osteoporosis [6-8]. Unfortunately, many adults who are obese also have sedentary lifestyles, a factor that further increases their risk for developing chronic illness. Therefore, promotion of physical activity is especially important for adults to help prevent the development of chronic illness.

Physical activity, defined as any movement that involves physical effort, is not synonymous with exercise but includes lifestyle activity. The current recommendation by the U.S. Department of Health is that adults obtain at least 150 minutes of physical activity of moderate-intensity activity or 75 minutes a week of vigorous-intensity activity each week [8]. However, another measure of adequate physical activity, walking at least 10,000 steps a day, is considered indicative of an "active" lifestyle [9]. Although data suggest that intense physical activity is most efficacious for increased health benefits, results from population-level studies suggest that even walking at a moderate pace has positive health benefits of decreased obesity, improved insulin resistance, and reduced mortality [10-12].

Findings from several studies using subjective measures of activity indicate that increased OSA severity is associated with decreased physical activity $[13,14]$. In addition, excessive daytime sleepiness, a frequent symptom of OSA, has been associated with both increased obesity and decreased functional activity in areas sensitive to sleep disruption [15]. However, it remains unclear if OSA or excessive daytime 
sleepiness affects objectively measured physical activity. The purpose of this study is to examine the relationship between subjective daytime sleepiness, OSA severity, functional activity, and objectively measured physical activity in order to obtain a multidimensional perspective of the association between nighttime sleep and daytime activity.

\section{Methods}

2.1. Participants. Participants were recruited from patients evaluated for sleep disorders at the University of Pittsburgh Medical Center Sleep Medicine Center. The sample was limited to individuals scheduled for an overnight sleep study for the evaluation of possible OSA. The eligibility criteria excluded persons already treated for OSA, coexistence of other sleep disorders such as insomnia or restless leg syndrome, regular use of sedative, hypnotic, or alerting medications, rotating shift or night-shift work, or pregnancy. The study had Institutional Review Board approval by the University of Pittsburgh. For this paper, we conducted analyses on baseline data from 37 participants having complete data on the study variables of interest.

2.2. Measures. Questionnaires developed at the University of Pittsburgh's School of Nursing were used to obtain data on sociodemographic factors including age, gender, race, marital status, years of formal education, and comorbid diseases. Weight was measured on a digital scale with the subject wearing light clothing and without shoes. Height was measured on a wall-mounted stadiometer. BMI was computed using the following formula: $\mathrm{BMI}=$ weight $(\mathrm{kg}) /$ height $\left(\right.$ meters $\left.^{2}\right)$.

Objective sleep disorders were determined by the diagnostic overnight polysomnography (PSG) to determine the presence and severity of suspected OSA. Scoring of the PSG was performed according to the criteria of American Academy of Sleep Medicine [16]. Apnea was defined as the complete cessation of airflow associated with a $4 \%$ oxygen desaturation that persisted for $>10$ seconds; hypopnea was a decreased airflow of at least 30\% associated with a drop of at least $4 \%$ in oxygen saturation. The PSG study recorded the electroencephalogram (EEG (C3A2 with C4A1 as backup, O2A1 with O1A2 as backup)), bilateral electrooculograms (EOG (right and left outer canthi referenced to A2 and A1)), bipolar submental and bilateral tibialis anterior electromyograms, chest and abdominal expansion by inductance plethysmography, airflow via nasal pressure and oral thermistor recording, pulse oximetry, and electrocardiogram. The apnea + hypopnea index (AHI) was the endpoint used to determine OSA severity.

Subjective daytime sleepiness was quantified by the Epworth Sleepiness Scale (ESS) [17], an 8-item questionnaire that asks the respondent to rate the possibility of their dozing in eight soporific situations. Sleepiness was graded on a 4point Likert scale ranging from 0 "no chance of dozing" to 3 "high chance of dozing". The ESS is a validated single-factor instrument with test-retest reliability $(r=.82)$ and internal consistency (Cronbach's alpha $=.88)$. At a cut-point of $\geq 10$, the ESS has a sensitivity of $93.5 \%$ and a specificity of $100 \%$ for distinguishing normal from pathological sleepiness [1820]. The total score on the ESS was the endpoint to determine subjective daytime sleepiness.

Functional activity was determined by the Functional Outcomes of Sleep Questionnaire (FOSQ) [21], a questionnaire designed to assess if sleepiness makes performing certain activities difficult. An example of FOSQ questions is "Do you have difficulty being as active as you want to be in the evening because you are sleepy or tired?" This 30-item questionnaire (Total Scale alpha $=.95$ ) contains five subscales: General Productivity (alpha $=.86$ ), Vigilance $($ alpha $=.87)$, Social Outcomes (alpha $=.88)$, Activity Level (alpha $=.91)$, and Intimacy and Sexual Relationships (alpha $=.87)$. Test-retest reliability for the total scale was $r=.90$, for subscales, $r=.81$ to .90 . The total score and subscale scores were used as endpoints in this analysis.

Objective activity and energy expenditure were evaluated with the Bodymedia SenseWear Pro Armband activity monitor. This activity monitor is a two-axial accelerometer device that measures the duration and intensity of activity. The activity monitor was worn on an adjustable Velcro armband worn on the right upper arm for a 7-day period. Subjects were instructed to wear the activity armbands aroundthe-clock except for showering or engaging in any activity that could make the activity monitor wet. The primary endpoint for activity was summarized on each subject as the mean daily activity counts for steps walked: additional endpoints include total estimated energy expenditure and mean metabolic equivalent units (MET).

2.3. Procedure. Human subjects' approval was obtained at the University of Pittsburgh for both the Sleep Disorders Registry and the current study. Patients at the sleep center are routinely asked if they are willing to participate in a Sleep Disorders Registry. People who consented to the registry were sent a letter explaining the study and informing them they would be contacted by telephone by study personnel. Over $30 \%$ of those people contacted $(n=119)$ by telephone screening were eligible and enrolled in the study. The primary reason people were excluded during phone screening was that the person had already been diagnosed with sleep apnea and treated with continuous positive airway treatment.

At the baseline visit, we obtained informed consent and measured subject's height and weight. The evaluations were conducted in the Center for Translational Research Center at the University of Pittsburgh. A prepaid mailer was given to the subjects to return their physical activity monitor and the questionnaires. Results of the overnight sleep study were obtained from the medical record.

2.4. Statistical Analyses. Data analyses were conducted using SPSS v. 17 (SPSS Inc., Chicago, IL). All continuous variables were checked for normality. Because some continuous variables were skewed, summary statistics were presented as the median and interquartile range (IQR) as well as the mean 
and standard deviation and as frequencies and percentages for categorical variables. Bivariate relations between continuous variables were examined with Pearson productmoment correlation for approximately bivariately normally distributed data. The Spearman rank-order correlation was used to examine the bivariate association between variables that were either ordinally scaled or with skewed distributions. Two sample $t$-test or Mann-Whitney $U$ test, if data were ordinally scaled or nonnormally distributed, were used to examine differences in age, AHI, BMI, and self-reported daytime sleepiness between men and women. Predictors for the hierarchical regression analyses for mean daily number of steps walked were selected based on previous associations of age, gender, and ESS with AHI and measures of physical activity [22-25]. Age, gender, and ESS were entered in the first block of the hierarchical regression analysis; AHI was then entered on the second block of the regression analysis.

\section{Results}

3.1. Sample Characteristics. The demographic characteristics of the sample $(N=37)$ are presented in Table 1. Overall, our sample was evenly distributed by gender, predominantly white, and middle age. Most subjects were married and well educated (median years of formal education $=16$ ) but only $54 \%$ of the sample was employed full- or part-time. The majority of the sample was overweight or obese. This sleep clinic-based sample had a high prevalence of both objective sleep disordered breathing and excessive daytime sleepiness. Over $78 \%$ of the sample had diagnosable OSA $(\mathrm{AHI} \geq 5)$ and $24 \%$ of the sample had severe OSA (AHI $\geq 30$ ). Excessive daytime sleepiness (ESS $\geq 10$ ) was reported by $41 \%$ of the sample. Analysis of mean Bodymedia activity data found that many people in the sample were sedentary $(40 \%, n=13)$ with $<5000$ steps walked on average daily and few (19\%, $n=6$ ) were active with $\geq 10,000$ steps/day.

3.2. Demographic Characteristics, Sleep, and Activity. There was no statistically significant difference in age, ESS scores, FOSQ total and subscale scores, or objectively measured activity levels between male and female subjects. Based on BMI, female subjects were typically more obese than male subjects (female: median $=38.95, \mathrm{IQR}=12.65$; male: median $=31.00, \mathrm{IQR}=5.40$; Mann-Whitney $U=68$, $P=.001)$. Male subjects in the study had more severe OSA based on AHI (median $=19.34, \mathrm{IQR}=52.43$ ) compared to female subjects (Median $=8.57, \mathrm{IQR}=6.68$ ), although there was no statistically significant difference in this small sample (Mann-Whitney $U=157, P=.690$ ). Females in the sample were less active (median daily steps $=4195, \mathrm{IQR}=$ 4832 ) than males (median daily steps $=7352, \mathrm{IQR}=6004)$; however, it was again not statistically significant (MannWhitney $U=87, P=.13$ ).

Age and BMI were not significantly associated with subjective sleepiness (ESS), OSA severity (AHI), or subjective activity (FOSQ total and subscale scores). Results of correlational analyses using Spearman rank-order correlations suggest that increased age is associated with decreased
TABle 1: Demographic and clinical characteristics of sample $(N=37)$.

\begin{tabular}{|c|c|}
\hline Characteristics & $\begin{array}{l}\text { Measures of central tendency } \\
\text { dispersion, or frequency }\end{array}$ \\
\hline Age (years) & $\begin{array}{l}\text { Median }=51.00, \mathrm{IQR}=15 \\
\text { Mean }=49.51 \pm 11.45\end{array}$ \\
\hline Male $\%(n)$ & $53(19)$ \\
\hline White $\%(n)$ & $78(29)$ \\
\hline Married/living with partner\% $(n)$ & $62(23)$ \\
\hline Education $>12$ years $\%(n)$ & $76(28)$ \\
\hline Body Mass Index $\left(\mathrm{kg} / \mathrm{m}^{2}\right)$ & $\begin{array}{l}\text { Median }=32.30, \mathrm{IQR}=10.90 \\
\text { Mean }=33.98 \pm 7.35,\end{array}$ \\
\hline Epworth Sleepiness Scale (ESS) & Mean $=9.43 \pm 4.94$ \\
\hline $\begin{array}{l}\text { Functional Outcomes of Sleep } \\
\text { Questionnaire (FOSQ) Total Score }\end{array}$ & Median = 17.66, IQR = 5.87, \\
\hline Apnea + hypopnea index (AHI) & $\begin{array}{l}\text { Median }=9.94, \mathrm{IQR}=27.80 \\
\text { Mean }=21.74 \pm 25.40\end{array}$ \\
\hline Steps walked daily & $\begin{array}{l}\text { Median }=5881, \mathrm{IQR}=5896 \\
\text { Mean }=6,988 \pm 3,852\end{array}$ \\
\hline
\end{tabular}

physical activity as measured by mean number of steps walked per day $\left(r_{s}=-.35, P=.051\right)$ and decreased total daily energy expenditure $\left(r_{s}=-.47, P=.007\right)$. Although BMI was not significantly associated with the mean number of steps walked, an increase in BMI was associated with decreased activity intensity (mean MET, $r_{s}=-.48, P=$ .006).

3.3. Daytime Sleepiness, OSA Severity, and Activity. Table 2 reports the results of correlation analyses examining the relationships between daytime sleepiness, OSA severity, functional activity, and objectively measured activity. Excessive sleepiness was associated with increased difficulty to conduct normal activities measured by all five FOSQ subscale scores (General Productivity, Social Outcomes, Activity Level, Vigilance, and Intimacy and Sexual Relationships) and the FOSQ total score. No statistically significant associations were found between daytime sleepiness and average daily measures of objective physical activity (mean steps walked, total energy expenditure, or average METS). Difficulties in performing functional activities because of sleepiness (FOSQ total and subscale scores) were not significantly associated with any objective measure of physical activity.

OSA severity was not statistically significantly associated with either daytime sleepiness or functional activity (FOSQ total or subscale scores). Data suggest that increased OSA severity may be associated with decreased physical activity. There were negative associations between AHI and total energy expenditure $\left(r_{s}=-.32, P=.07\right)$, steps walked $\left(r_{s}=-.29, P=.11\right)$, and average METS $\left(r_{s}=-.29, P=.10\right)$ that approached statistical significance.

A multiple regression model (Table 3) with the daily mean number of steps as the dependent variable, controlling for gender, age, ESS and AHI, was found to be statistically significant overall $\left(F=3.91, R^{2}=.38, P=.01\right)$. 
TABLE 2: Correlations among study variables.

\begin{tabular}{lll}
\hline Variable pairs & Spearman's $r_{s}$ & $P$ value \\
\hline Mean steps and AHI & -.29 & .11 \\
ESS and total FOSQ & -.73 & $<.001$ \\
ESS and FOSQ General & -.67 & $<.001$ \\
Productivity & & $<.001$ \\
ESS and FOSQ Social Outcomes & -.63 & $<.001$ \\
ESS and FOSQ Activity Level & -.62 & $<.001$ \\
ESS and FOSQ Vigilance & -.80 & .001 \\
ESS and FOSQ Intimacy and & -.80 & \\
Sexual Relationships & &
\end{tabular}

TABLE 3: Regression results of objective physical activity (mean daily steps walked).

\begin{tabular}{lcc}
\hline Variable & Beta & $P$ value \\
\hline Age & -98.81 & .07 \\
Gender (male) & 3434.13 & .01 \\
Epworth & -110.07 & .35 \\
AHI & -64.25 & .01 \\
\hline
\end{tabular}

$R^{2}=.38$ (Adjusted $R^{2}=.28$ ).

The strongest predictors of number of steps were gender $(t=2.79, P=.01)$ and AHI $(t=-2.72, P=.01)$. Age approached statistical significance $(t=-1.90, P=.07)$. ESS was not statistically significantly associated $(t=-.95$, $P=.35$ ) with number of steps. An extra year of age was estimated to translate to a decrease of 98.81 steps per day. An extra point in AHI was found to result in a drop of 64.25 in mean daily steps. Male gender in this multiple regression analysis was associated with an additional 3434 steps per day.

\section{Discussion}

We found that increased OSA severity was associated with decreased objectively measured physical activity while controlling for age, gender, and daytime sleepiness (Table 3 ). In addition, while complaints of subjective sleepiness were strongly associated with increased self-reported difficulty in being active, there was no significant association between either sleepiness or self-reported difficulty because of sleepiness in performing functional activity and objectively measured activity.

The United States Department of Health and Human Services has set 150 minutes a week of moderate-intensity activity or 10,000 steps a day of walking as the goal [8]. Although the 2008 Physical Activities Guidelines and other study acknowledge that the goal of 10,000 steps a day may not be sustainable with elderly persons or persons with chronic illness, it is considered appropriate for most adults [7-9]. Increased activity is especially beneficial in persons who are moving from middle age into old age to promote healthy aging. In this study we found that OSA, a treatable sleep disorder, is associated with more sedentary lifestyles. Assessment and treatment of OSA is important not only because it is a harmful disease, but also because of its mediating effect on physical activity, an essential part of every adults program for healthy aging.

The sample (Table 1) of middle-aged, obese, sedentary persons is fairly representative of the aging population. The results of our study (Table 2) are consistent with previous research showing a significant association between decreased moderate to moderately vigorous physical activity and increased severity of OSA in the Sleep Heart Health Study (SHHS $(N=4275)$ ) [14]. The evaluation of physical activity in the SHHS was based on self-report, which might have affected the measurement of physical activity. In addition, the SHHS used different instruments for the assessment of physical activity in the five cohorts in the study which might have interfered with the precision of the findings. Our findings are important because they validate this relationship using an objective measurement of physical activity.

The finding that women are likely to be more obese and have less severe OSA than men is consistent with previous research among patients being evaluated in a sleep clinic for possible sleep apnea [22-26]. Women in the study were more likely to be sedentary than men, again a finding that agrees with the results of previous studies that utilized questionnaires to evaluate physical activity [22]. Although premenopausal women have a decreased risk for OSA, the risk of OSA increases in both men and women as they age. Unfortunately, OSA in women is frequently underdiagnosed because of a common misconception that OSA is a "male" disorder [27, 28].

The FOSQ is a validated questionnaire that has been used in multiple clinical trials to assess the impact of sleepiness on the ability to conduct daily activities [29-32]. The FOSQ questionnaire evaluates the degree of difficulty caused by sleepiness on functional outcomes such as being active and productive, able to maintain vigilance during a task like driving, watching a movie, being active in the morning/afternoon/evening, or being interested in engaging in intimate relationships. It is important to remember that the FOSQ was not developed as an indirect measure of physical activity. The strong association between daytime sleepiness on the Epworth Sleepiness Scale and the FOSQ (Table 2) might have resulted because the instruments measure subjective difficulty rather than objective behavior. This incongruity between objective physical activity and subjective report of difficulty in performing functional activity is similar to the well-known discordance between self-reports of medication adherence and objective medication behavior [33].

There are several limitations of the present study. Since this study had a cross-sectional observational design, we can only report associations and cannot infer causation. Sleepiness was evaluated only by subjective self-assessment by subjects and there was no objective assessment of excessive sleepiness such as with a multiple sleep latency test. Therefore, future evaluation of the effect of objective sleepiness on physical activity is warranted. The small sample size and resultant lack of statistical power resulted in several tests possibly having a type- 2 error. Because there was a small convenience sample that consisted exclusively of sleep clinic patients, the findings of this study need to be considered 
as preliminary and cannot be generalized to the general adult population. Finally, although we attempted to control potential confounders, we cannot dismiss the possibility that important variables might not have been included in the analyses.

\section{Conclusions}

An active lifestyle with regular physical activity is essential for health promotion and disease prevention for healthy aging. While exercise of longer duration and greater intensity is optimal, an active lifestyle is fundamental to health promotion across the lifespan. Although previous studies found that sleepiness and OSA were associated with decreased self-reported activity, this study's utilization of an objective activity monitor helped quantify the effect of OSA severity and sleepiness on physical activity.

Evidence from previous studies has strongly implicated the role of daytime sleepiness in negatively affecting participation in functional activities [13, 15]. Excessive daytime sleepiness is not benign, and as also found in the current study, has a negative effect on quality of life. Because excessive sleepiness is nonspecific, a comprehensive evaluation of this important symptom is important for health promotion and disease prevention in the aging adult. This is relevant for both men and women because of the importance of physical activity for healthy aging and the implication that untreated OSA may negatively impact the ability to have an active lifestyle.

\section{Authors' Contributions}

E. Chasens is responsible for concept and design, interpretation of data, and preparation of manuscript. S. Sereika is responsible for interpretation of data, preparation of manuscript. M. Houze is responsible for interpretation of data and writing of results. P. Strollo reformed medical management, interpretation of data, and preparation of manuscript.

\section{Acknowledgment}

The funding for this project came from grant support received from the NIH/NCRR/CTSA Grant UL1 RR024153 and the University of Pittsburgh Central Research Development Fund.

\section{References}

[1] T. Young, P. E. Peppard, and S. Taheri, "Excess weight and sleep-disordered breathing," Journal of Applied Physiology, vol. 99, no. 4, pp. 1592-1599, 2005.

[2] American Diabetes Association, "Diagnosis and classification of diabetes," Diabetes Care, vol. 29, no. 1, pp. S43-S48, 2006.

[3] S. M. Grundy, J. I. Cleeman, S. R. Daniels et al., "Diagnosis and management of the metabolic syndrome," Circulation, vol. 112, no. 17, pp. 2735-2752, 2005.

[4] J. E. Shaw, N. M. Punjabi, J. P. Wilding, K. G. M. M. Alberti, and P. Z. Zimmet, "Sleep-disordered breathing and type 2 diabetes. A report from the International Diabetes Federation Taskforce on Epidemiology and Prevention," Diabetes Research and Clinical Practice, vol. 81, no. 1, pp. 2-12, 2008.

[5] V. K. Somers, D. P. White, R. Amin et al., "Sleep Apnea and Cardiovascular Disease: An American Heart Association/American College of Cardiology Foundation scientific statement from the American Heart Association Council for High Blood Pressure Research Professional Education Committee, Council on Clinical Cardiology, Stroke Council, and Council on Cardiovascular Nursing," Circulation, vol. 118, no. 10, pp. 1080-1111, 2008.

[6] F. B. Hu, M. J. Stampfer, C. Solomon et al., "Physical activity and risk for cardiovascular events in diabetic women," Annals of Internal Medicine, vol. 134, no. 2, pp. 96-105, 2001.

[7] A. Kriska, "Physical activity and the prevention of type 2 diabetes mellitus: how much for how long?" Sports Medicine, vol. 29, no. 3, pp. 147-151, 2000.

[8] U.S. Department of Health and Human Services, 2008 Physical Activity Guidelines for Americans, U.S. Department of Health and Human Services, Washington, DC, USA, 2008.

[9] C. Tudor-Locke and D. R. Bassett Jr., "How many steps/day are enough? Preliminary pedometer indices for public health," Sports Medicine, vol. 34, no. 1, pp. 1-8, 2004.

[10] E. W. Gregg, R. B. Gerzoff, C. J. Caspersen, D. F. Williamson, and K. M. V. Narayan, "Relationship of walking to mortality among US adults with diabetes," Archives of Internal Medicine, vol. 163, no. 12, pp. 1440-1447, 2003.

[11] F. B. Hu, R. J. Sigal, J. W. Rich-Edwards et al., "Walking compared with vigorous physical activity and risk of type 2 diabetes in women: a prospective study," Journal of the American Medical Association, vol. 282, no. 15, pp. 1433-1439, 1999.

[12] A. Morabia and M. C. Costanza, "Does walking 15 minutes per day keep the obesity epidemic away? Simulation of the efficacy of a populationwide campaign," American Journal of Public Health, vol. 94, no. 3, pp. 437-440, 2004.

[13] E. R. Chasens, M. G. Umlauf, and T. E. Weaver, "Sleepiness, physical activity, and functional outcomes in veterans with type 2 diabetes," Applied Nursing Research, vol. 22, no. 3, pp. 176-182, 2009.

[14] S. F. Quan, G. T. O’Connor, J. S. Quan et al., "Association of physical activity with sleep-disordered breathing," Sleep and Breathing, vol. 11, no. 3, pp. 149-157, 2007.

[15] E. R. Chasens, S. M. Sereika, T. E. Weaver, and M. G. Umlauf, "Daytime sleepiness, exercise, and physical function in older adults," Journal of Sleep Research, vol. 16, no. 1, pp. 60-65, 2007.

[16] C. Iber, S. Ancoli-Israel, A. Chesson, and S. F. Quan, The AASM Manual for the Scoring of Sleep and Associated Events: Rules, Terminology and Technical Specifications, American Academy of Sleep Medicine, Westchester, Ill, USA, 2007.

[17] M. W. Johns, "A new method for measuring daytime sleepiness: the Epworth sleepiness scale," Sleep, vol. 14, no. 6, pp. 540-545, 1991.

[18] M. W. Johns, "Reliability and factor analysis of the Epworth Sleepiness Scale," Sleep, vol. 15, no. 4, pp. 376-381, 1992.

[19] M. W. Johns, "Daytime sleepiness, snoring, and obstructive sleep apnea; The Epworth Sleepiness Scale," Chest, vol. 103, no. 1, pp. 30-36, 1993.

[20] M. W. Johns, "Sensitivity and specificity of the multiple sleep latency test (MSLT), the maintenance of wakefulness test and the Epworth sleepiness scale: failure of the MSLT as a gold standard," Journal of Sleep Research, vol. 9, no. 1, pp. 5-11, 2000 . 
[21] T. E. Weaver, A. M. Laizner, L. K. Evans et al., "An instrument to measure functional status outcomes for disorders of excessive sleepiness," Sleep, vol. 20, no. 10, pp. 835-843, 1997.

[22] M. Basta, H. M. Lin, S. Pejovic, A. Sarrigiannidis, E. O. Bixler, and A. N. Vgontzas, "Lack of regular exercise, depression, and degree of apnea are predictors of excessive daytime sleepiness in patients with sleep apnea: sex differences," Journal of Clinical Sleep Medicine, vol. 4, no. 1, pp. 19-25, 2008.

[23] E. O. Bixler, A. N. Vgontzas, H. M. Lin, S. L. Calhoun, A. VelaBueno, and A. Kales, "Excessive daytime sleepiness in a general population sample: the role of sleep apnea, age, obesity, diabetes, and depression," Journal of Clinical Endocrinology and Metabolism, vol. 90, no. 8, pp. 4510-4515, 2005.

[24] D. J. Gottlieb, C. W. Whitney, W. H. Bonekat et al., "Relation of sleepiness to respiratory disturbance index: the sleep heart health study," American Journal of Respiratory and Critical Care Medicine, vol. 159, no. 2, pp. 502-507, 1999.

[25] M. L. Unruh, S. Redline, M. W. An et al., "Subjective and objective sleep quality and aging in the sleep heart health study," Journal of the American Geriatrics Society, vol. 56, no. 7, pp. 1218-1227, 2008.

[26] T. Young, J. Skatrud, and P. E. Peppard, "Risk factors for obstructive sleep apnea in adults," Journal of the American Medical Association, vol. 291, no. 16, pp. 2013-2016, 2004.

[27] T. Young, R. Hutton, L. Finn, S. Badr, and M. Palta, "The gender bias in sleep apnea diagnosis: are women missed because they have different symptoms?" Archives of Internal Medicine, vol. 156, no. 21, pp. 2445-2451, 1996.

[28] T. Young, M. Palta, J. Dempsey, J. Skatrud, S. Weber, and S. Badr, "The occurrence of sleep-disordered breathing among middle-aged adults," The New England Journal of Medicine, vol. 328, no. 17, pp. 1230-1235, 1993.

[29] M. Barnes, D. Houston, C. J. Worsnop et al., "A randomized controlled trial of continuous positive airway pressure in mild obstructive sleep apnea," American Journal of Respiratory and Critical Care Medicine, vol. 165, no. 6, pp. 773-780, 2002.

[30] J. F. Faccenda, T. W. Mackay, N. A. Boon, and N. J. Douglas, "Randomized placebo-controlled trial of continuous positive airway pressure on blood pressure in the sleep apneahypopnea syndrome," American Journal of Respiratory and Critical Care Medicine, vol. 163, no. 2, pp. 344-348, 2001.

[31] J. M. Montserrat, M. Ferrer, L. Hernandez et al., "Effectiveness of CPAP treatment in daytime function in sleep apnea syndrome: a randomized controlled study with an optimized placebo," American Journal of Respiratory and Critical Care Medicine, vol. 164, no. 4, pp. 608-613, 2001.

[32] R. D. Wells, K. E. Freedland, R. M. Carney, S. P. Duntley, and E. J. Stepanski, "Adherence, reports of benefits, and depression among patients treated with continuous positive airway pressure," Psychosomatic Medicine, vol. 69, no. 5, pp. 449-454, 2007.

[33] J. Dunbar-Jacob and M. K. Mortimer-Stephens, "Treatment adherence in chronic disease," Journal of Clinical Epidemiology, vol. 54, no. 12, supplement 1, pp. S57-S60, 2001. 


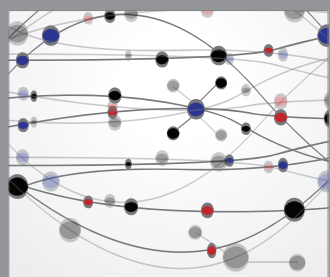

The Scientific World Journal
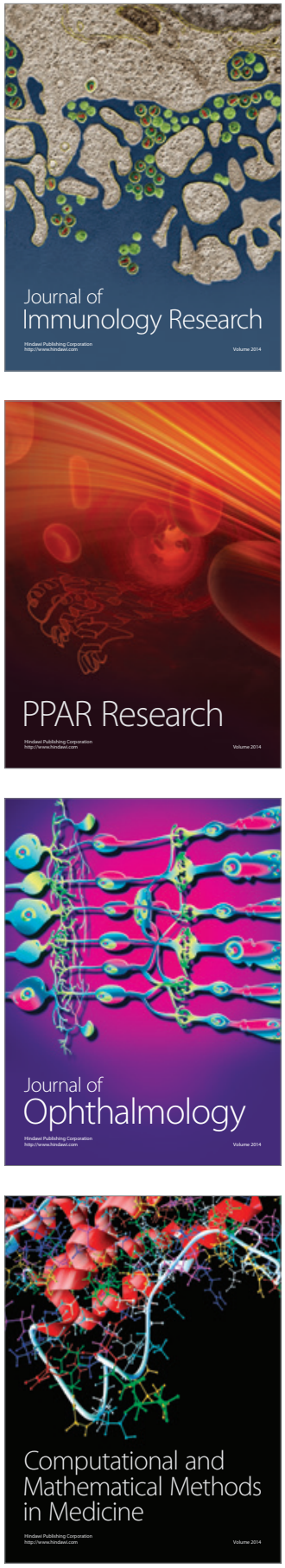

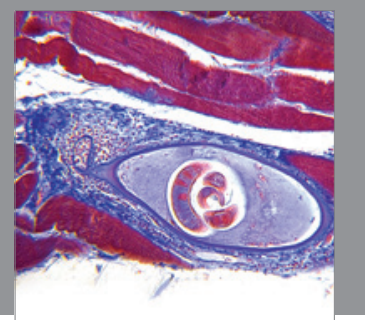

Gastroenterology

Research and Practice
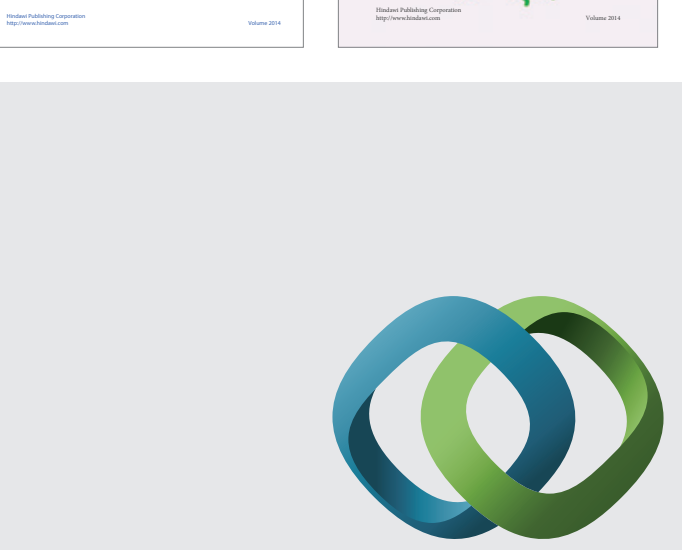

\section{Hindawi}

Submit your manuscripts at

http://www.hindawi.com
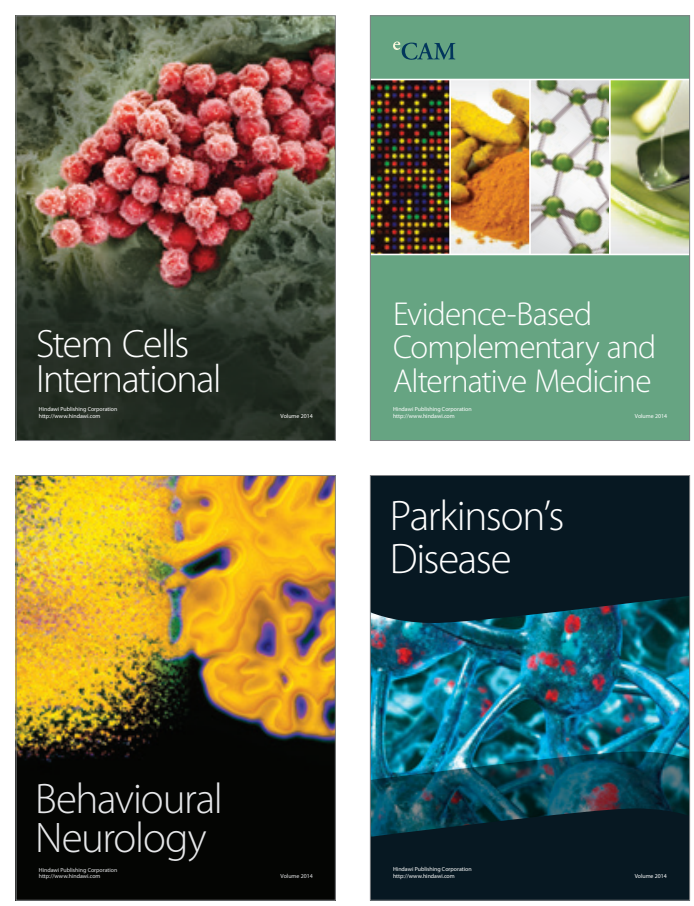

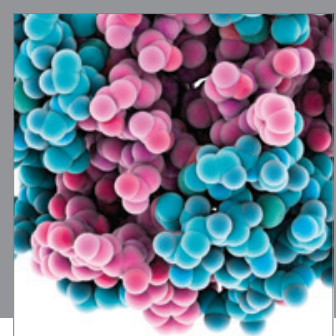

Journal of
Diabetes Research

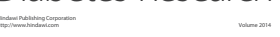

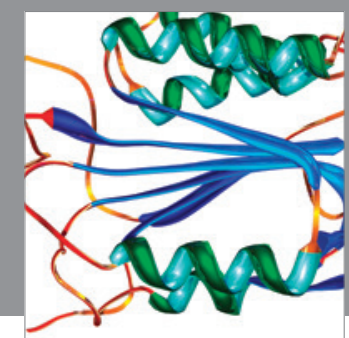

Disease Markers
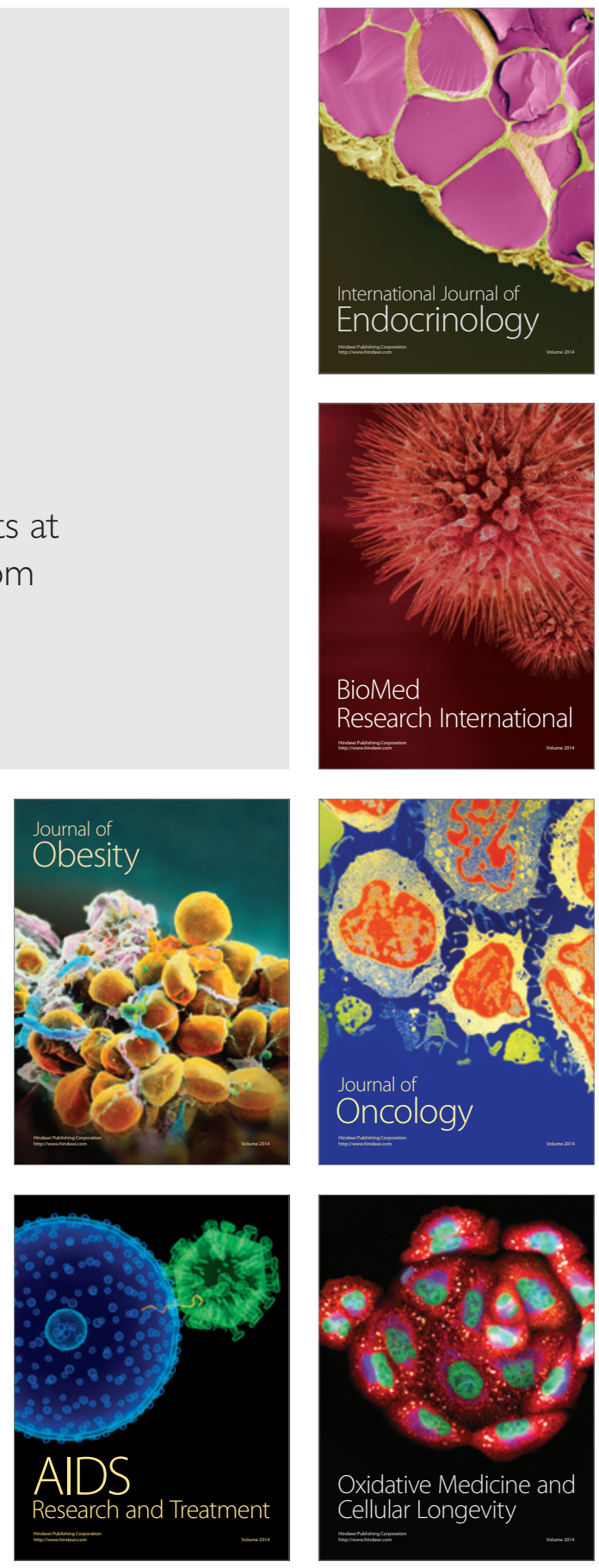